Xifeng Yan, Shibin Zhong* and Hong Zhang

\title{
The crystal structure of $(E)-N^{\prime}-(1-(3-c h l o r o-4-$ fluorophenyl) ethylidene)-2-hydroxy- benzohydrazide, $\mathrm{C}_{15} \mathrm{H}_{12} \mathrm{ClFN}_{2} \mathrm{O}_{2}$
}
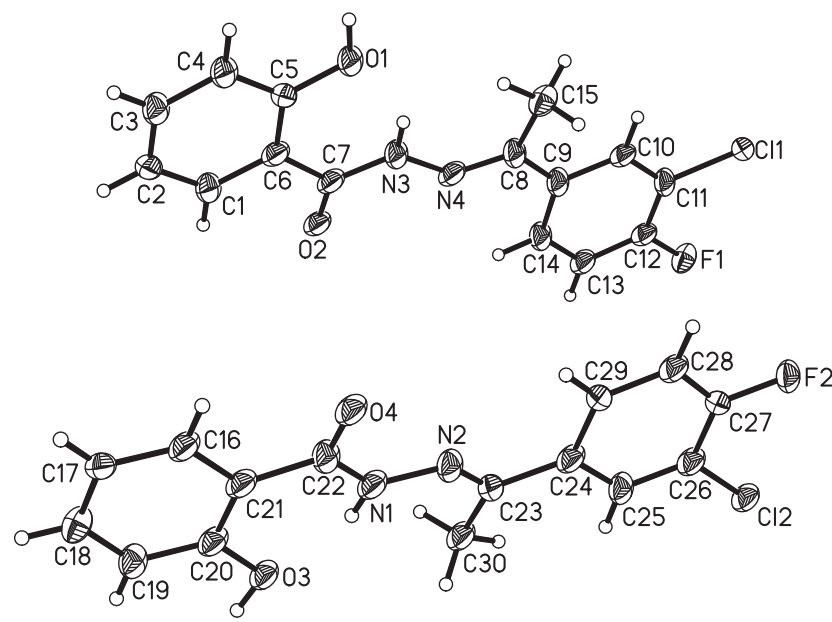

https://doi.org/10.1515/ncrs-2019-0571

Received August 7, 2019; accepted October 8, 2019; available online October 25, 2019

\section{Abstract \\ $\mathrm{C}_{15} \mathrm{H}_{12} \mathrm{ClFN}_{2} \mathrm{O}_{2}$, monoclinic, $P 2_{1} / c$ (no. 14), $a=13.9210$ (13) $\AA$, $b=13.2329(15) \AA, \quad c=18.7191(19) \AA, \quad \beta=126.250(2)^{\circ}, \quad V=$ $2780.9(5) \AA^{3}, \quad Z=8, \quad R_{\mathrm{gt}}(F)=0.0499, \quad w R_{\mathrm{ref}}\left(F^{2}\right)=0.1420$, $T=293(2) \mathrm{K}$}

\section{CCDC no.: 1958013}

The asymmetric unit of the molecular structure is shown in the figure. Table 1 contains crystallographic data and Table 2 contains the list of the atoms including atomic coordinates and displacement parameters.

*Corresponding author: Shibin Zhong, Jiangxi University of Science and Technology, School of Architectural and Surverying and Mapping Engineering, 341000 Ganzhou, Jiangxi, P.R. China; and Jiangxi Provincial Archives of Natural Resources, 330025 Nanchang, Jiangxi, P.R. China, e-mail: shibinzhong@163.com. https://orcid.org/0000-0001-9203-7959

Xifeng Yan: East China University of Technology, School of Water Resources and Enviromental Engineering, 330013 Nanchang, Jiangxi, P.R. China

Hong Zhang: East China 263 Engineering Survey Institute of Nuclear Industry, 343100 Ji'an, Jiangxi, P.R. China
Table 1: Data collection and handling.

\begin{tabular}{ll}
\hline Crystal: & Colourless block \\
Size: & $0.20 \times 0.15 \times 0.12 \mathrm{~mm}$ \\
Wavelength: & Mo $K \alpha$ radiation $(0.71073 \AA)$ \\
$\mu:$ & $0.29 \mathrm{~mm}^{-1}$ \\
Diffractometer, scan mode: & Bruker APEX-II, $\varphi$ and $\omega$ \\
$\theta_{\text {max }}$, completeness: & $27.5^{\circ},>99 \%$ \\
$N\left(h k l_{\text {measured }}, N(h k l)_{\text {unique }}, R_{\text {int }}:\right.$ & $19612,6361,0.084$ \\
Criterion for $I_{\text {obs }}, N(h k l)_{\mathrm{gt}}:$ & $I_{\text {obs }}>2 \sigma\left(I_{\text {obs }}\right), 4081$ \\
$N(\text { param })_{\text {refined }}:$ & 381 \\
Programs: & Bruker $[1]$, SHELX [2] \\
\hline
\end{tabular}

Table 2: Fractional atomic coordinates and isotropic or equivalent isotropic displacement parameters $\left(\AA^{2}\right)$.

\begin{tabular}{lrrrr}
\hline Atom & $\boldsymbol{X}$ & $\boldsymbol{y}$ & $\boldsymbol{Z}$ & $\boldsymbol{U}_{\text {iso }}{ }^{*} \boldsymbol{U}_{\text {eq }}$ \\
\hline C1 & $1.0604(2)$ & $0.1301(2)$ & $0.87188(19)$ & $0.0507(7)$ \\
H1 & 1.0593 & 0.1998 & 0.8651 & $0.061^{*}$ \\
C2 & $1.0925(2)$ & $0.0916(2)$ & $0.95159(19)$ & $0.0513(7)$ \\
H2 & 1.1138 & 0.1346 & 0.9982 & $0.062^{*}$ \\
C3 & $1.0924(2)$ & $-0.0121(2)$ & $0.96110(19)$ & $0.0514(7)$ \\
H3 & 1.1128 & -0.0391 & 1.0143 & $0.062^{*}$ \\
C4 & $1.0626(2)$ & $-0.0756(2)$ & $0.89280(18)$ & $0.0513(7)$ \\
H4 & 1.0640 & -0.1451 & 0.9004 & $0.062^{*}$ \\
C5 & $1.0304(2)$ & $-0.0370(2)$ & $0.81273(18)$ & $0.0497(7)$ \\
C6 & $1.0294(2)$ & $0.06784(19)$ & $0.80076(18)$ & $0.0504(7)$ \\
C7 & $0.9952(2)$ & $0.1184(2)$ & $0.71837(18)$ & $0.0502(7)$ \\
C8 & $0.8638(2)$ & $0.0364(2)$ & $0.49458(19)$ & $0.0525(8)$ \\
C9 & $0.8362(2)$ & $0.0955(2)$ & $0.41578(18)$ & $0.0505(7)$ \\
C10 & $0.8011(2)$ & $0.0419(2)$ & $0.33914(18)$ & $0.0510(7)$ \\
H10 & 0.8037 & -0.0283 & 0.3409 & $0.061^{*}$ \\
C11 & $0.7634(2)$ & $0.08997(19)$ & $0.26193(18)$ & $0.0494(7)$ \\
C12 & $0.7603(2)$ & $0.1938(2)$ & $0.25817(19)$ & $0.0514(7)$ \\
C13 & $0.7950(2)$ & $0.2521(2)$ & $0.33139(18)$ & $0.0533(7)$ \\
H13 & 0.7933 & 0.3223 & 0.3287 & $0.064^{*}$ \\
C14 & $0.8322(2)$ & $0.20163(19)$ & $0.40882(19)$ & $0.0538(8)$ \\
H14 & 0.8557 & 0.2397 & 0.4585 & $0.065^{*}$ \\
C15 & $0.8372(2)$ & $-0.07834(19)$ & $0.49145(18)$ & $0.0527(7)$ \\
H15A & 0.8685 & -0.1016 & 0.5500 & $0.079^{*}$ \\
H15B & 0.8741 & -0.1150 & 0.4693 & $0.079^{*}$ \\
H15C & 0.7527 & -0.0892 & 0.4531 & $0.079^{*}$ \\
C16 & $0.5796(2)$ & $0.14467(19)$ & $0.87763(18)$ & $0.0494(7)$ \\
H16 & 0.5765 & 0.0748 & 0.8714 & $0.059^{*}$ \\
C17 & $0.6282(2)$ & $0.18537(19)$ & $0.96035(19)$ & $0.0512(7)$
\end{tabular}


Table 2 (continued)

\begin{tabular}{lrrrr}
\hline Atom & $\boldsymbol{x}$ & $\boldsymbol{y}$ & $\boldsymbol{z}$ & $\boldsymbol{U}_{\text {iso }} / \boldsymbol{U}_{\text {eq }}$ \\
\hline H17 & 0.6581 & 0.1437 & 1.0093 & $0.061^{*}$ \\
C18 & $0.6317(2)$ & $0.28978(19)$ & $0.96899(18)$ & $0.0487(7)$ \\
H18 & 0.6653 & 0.3184 & 1.0244 & $0.058^{*}$ \\
C19 & $0.5861(2)$ & $0.3513(2)$ & $0.89660(18)$ & $0.0511(7)$ \\
H19 & 0.5871 & 0.4210 & 0.9032 & $0.061^{*}$ \\
C20 & $0.5385(2)$ & $0.31049(19)$ & $0.81365(18)$ & $0.0483(7)$ \\
C21 & $0.5347(2)$ & $0.20525(19)$ & $0.80263(18)$ & $0.0489(7)$ \\
C22 & $0.4876(2)$ & $0.1529(2)$ & $0.71805(18)$ & $0.0496(7)$ \\
C23 & $0.3919(2)$ & $0.2306(2)$ & $0.50090(19)$ & $0.0532(8)$ \\
C24 & $0.3444(2)$ & $0.16985(19)$ & $0.41862(18)$ & $0.0500(7)$ \\
C25 & $0.2960(2)$ & $0.2210(2)$ & $0.33834(17)$ & $0.0510(7)$ \\
H25 & 0.2888 & 0.2909 & 0.3370 & $0.061^{*}$ \\
C26 & $0.2592(2)$ & $0.17142(19)$ & $0.26227(18)$ & $0.0504(7)$ \\
C27 & $0.2680(2)$ & $0.06791(19)$ & $0.26216(18)$ & $0.0497(7)$ \\
C28 & $0.3124(2)$ & $0.0105(2)$ & $0.33861(18)$ & $0.0509(7)$ \\
H28 & 0.3163 & -0.0597 & 0.3382 & $0.061^{*}$ \\
C29 & $0.3502(2)$ & $0.06377(19)$ & $0.41518(18)$ & $0.0510(7)$ \\
H29 & 0.3810 & 0.0274 & 0.4670 & $0.061^{*}$ \\
C30 & $0.4129(2)$ & $0.34686(19)$ & $0.50572(18)$ & $0.0559(8)$ \\
H30A & 0.3633 & 0.3804 & 0.5183 & $0.084^{*}$ \\
H30B & 0.3932 & 0.3703 & 0.4500 & $0.084^{*}$ \\
H30C & 0.4950 & 0.3615 & 0.5518 & $0.084^{*}$ \\
C11 & $0.71888(6)$ & $0.02090(5)$ & $0.16886(5)$ & $0.0551(2)$ \\
C12 & $0.20314(5)$ & $0.23827(5)$ & $0.16556(4)$ & $0.0528(2)$ \\
F1 & $0.72159(12)$ & $0.24023(11)$ & $0.18107(10)$ & $0.0539(4)$ \\
F2 & $0.23185(12)$ & $0.01976(11)$ & $0.18642(10)$ & $0.0520(4)$ \\
N1 & $0.45966(17)$ & $0.21313(16)$ & $0.64992(15)$ & $0.0488(6)$ \\
H1A & 0.4694 & 0.2772 & 0.6590 & $0.059^{*}$ \\
N2 & $0.41626(17)$ & $0.17763(16)$ & $0.56630(15)$ & $0.0497(6)$ \\
N3 & $0.95095(17)$ & $0.05715(16)$ & $0.64765(15)$ & $0.0493(6)$ \\
H3A & 0.9464 & -0.0064 & 0.6547 & $0.059^{*}$ \\
N4 & $0.91240(18)$ & $0.09048(16)$ & $0.56430(15)$ & $0.0519(6)$ \\
O1 & $1.00053(14)$ & $-0.09903(13)$ & $0.74459(12)$ & $0.0511(5)$ \\
H1E & 1.0059 & -0.1680 & 0.7625 & $0.077^{*}$ \\
\hline & $1.00823(14)$ & $0.21007(13)$ & $0.71545(12)$ & $0.0520(5)$ \\
O3E & $0.49501(14)$ & $0.37106(12)$ & $0.74203(12)$ & $0.0500(5)$ \\
O.47437(15) & $0.06042(13)$ & $0.71121(12)$ & $0.0533(5)$ \\
\hline
\end{tabular}

\section{Source of material}

A mixture of 2-hydroxy-benzoic acid hydrazide (1 mmol, $152.2 \mathrm{mg}$ ) and 1-(3-chloro-4-fluoro-phenyl)-ethanone (1 mmol, $172.6 \mathrm{mg}$ ) in anhydrous ethanol (25 mL) was kept under reflux conditions $(351 \mathrm{~K})$ for $3 \mathrm{~h}$. The solvent was removed under reduced pressure and the solid product was recrystallized from $15 \mathrm{~mL}$ of anhydrous ethanol. After 4 days, colourless block-shaped crystals were obtained.

\section{Experimental details}

Coordinates of hydrogen atoms were refined without any constraints or restraints. The $U_{\text {iso }}$ values were set to be $1.5 U_{\text {eq }}$ of the carrier atom for methyl $\mathrm{H}$ atoms and $1.2 U_{\text {eq }}$ for the remaining $\mathrm{H}$ atoms.

\section{Comment}

In recent years, Schiff bases as one of the important synthetic intermediates have attracted more and more attention due to the wide applications in the fields of coordination chemistry, biochemistry, pharmacy, nanotechnology, and photochemistry [3-10]. From the structural point of view, $o$-hydroxy Schiff bases are attractive because of their intramolecular $\mathrm{H}$-bond, and thus have been extensively investigated and reported [11-17]. In addition, the heteroatoms (such as N, F, $\mathrm{Cl}, \mathrm{Br}, \mathrm{S}$ ) can act as hydrogen bonding acceptors for bioactive molecules in order to exhibit higher biological activity $[18,19]$. But, the unsymmetrical $o$-hydroxy Schiff bases containing heteroatoms especial of $\mathrm{F}$ and $\mathrm{Cl}$ in one molecule are less investigated. Herein, in order to search for new $o$-hydroxy Schiff bases containing $\mathrm{F}$ and $\mathrm{Cl}$ heteroatoms, we have undertaken the synthesis and single crystal structure determination of the title compound ( $c f$. the figure).

In the title compound, there are two crystallographically independent molecules in the asymmetric unit, which have very similar conformations. Compared with those of closely related structure, such a structural feature is uncommon [15]. The bond lengths and angles are in the expected ranges which fit with those of related compounds in literature [11-17]. The $\mathrm{C}=\mathrm{N}$ double bond lengths in the independent molecule are 1.277(3) $\AA$ ( $\mathrm{C} 8=\mathrm{N} 4)$ and 1.271(4) $\AA$ (C23=N2), respectively, exhibiting the double bond character. The aromatic rings form dihedral angles of $3.9^{\circ}$ and $4.2^{\circ}$ in the two independent molecules. In the crystal structure, the molecules are linked into double infinite chains by $\mathrm{O}-\mathrm{H} \cdots \mathrm{O}$ hydrogen bonds. There also exist intramolecular $\mathrm{N}-\mathrm{H}$... O hydrogen bonds, which further consolidate the crystal packing.

Acknowledgements: This work was financially supported by East China University of Technology.

\section{References}

1. Bruker. APEX2, SAINT and SADABS. Bruker AXS Inc., Madison, WI, USA (2008).

2. Sheldrick, G. M.: A short history of SHELX. Acta Crystallogr. A64 (2008) 112-122.

3. Zhang, L. S.; Chen, Q.; Hou, G. G.; Zhao, W.; Hou, Y.: Hydroxyl-substituted double Schiff-base condensed 4piperidone/cyclohexanones as potential anticancer agents with biological evaluation. J. Enzyme Inhib. Med. Chem. 34 (2019) 264-271.

4. $\mathrm{Pu}, \mathrm{X} .:$ The crystal structure of $(E)-4$-chloro-2-(((5methylpyridin-2-yl)imino)methyl)phenol, $\mathrm{C}_{13} \mathrm{H}_{11} \mathrm{ClN}_{2} \mathrm{O}$. Z. Kristallogr. NCS 233 (2018) 253-254.

5. Chang, F. F.; Zhang, L.; Zhao, P. C.; Huang, W.: Transmetalation for flexible pendant-armed Schiff-base macrocyclic complexes influenced by halide effects. Inorg. Chem. 57 (2018) 1438-1448. 
6. Aboura, W.; Benabdallah, T.; Zhang, F.; Therrien, B.: Alkoxylation of the imine carbon atom of a Schiff-base ligand upon coordination to arene ruthenium. Inorg. Chim. Acta 483 (2018) 93-97.

7. Kadwa, E.; Bala, M. D.; Friedrich, H. B.: Base metal Schiff base complexes applied as catalysts for the oxidation of $n$-octane. Inorg. Chim. Acta 463 (2017) 112-117.

8. Shanty, A.; Philip, J.; Sneha, E.; Kurup, M.; Balachandran, S.; Mohanan, P.: Synthesis, characterization and biological studies of Schiff bases derived from heterocyclic moiety. Bioorg. Chem. 70 (2017) 67-73.

9. Abu-Dief, A. M.; Mohamed, I. M. A.: A review on versatile applications of transition metal complexes incorporating Schiff bases. Beni-suef Univ. J. Basic Appl. Sci. 4 (2015) 119-133.

10. Li, P.; Niu, M.-J.; Hong, M.; Cheng, S.; Dou, J.-M.: Effect of structure and composition of nickel(II) complexes with salicylidene Schiff base ligands on their DNA/protein interaction and cytotoxicity. J. Inorg. Biochem. 137 (2014) 101-108.

11. Fan, C. G.; Song, M. Z.: (E)- $N^{\prime}$-[1-(4-Bromophenyl)ethylidene]-2hydroxybenzohydrazide. Acta Crystallogr. E65 (2009) 02792.

12. Li, M. L.; Huang, X.; Feng, R. K.: (E)-N'-[1-(4-Chlorophenyl) ethylidene]-2-hydroxybenzohydrazide. Acta Crystallogr. E65 (2009) 0369.

13. Yue, S. Y.; Lu, J. F.: $N^{\prime}$-[1-(2-Hydroxyphenyl)ethylidene]-2methoxybenzohydrazide. Acta Crystallogr. E66 (2010) 01550.
14. Qiu, X. Y.; Luo, Q. Y.; Yang, S. L.; Liu, W. S.: (E)-2-Hydroxy$N^{\prime}$-[1-(4-methoxyphenyl)-ethylidene]benzohydrazide. Acta Crystallogr. E62 (2006) 04291-04292.

15. Patra, D.; Biswas, N.; Kumari, B.; Das, P.; Sepay, N.; Chatterjee, S.; Drew, M. G. B.; Ghosh, T.: A family of mixed-ligand oxidovanadium(V) complexes with aroylhydrazone ligands: a combined experimental and computational study on the electronic effects of para substituents of hydrazone ligands on the electronic properties, DNA binding and nuclease activities. RSC Adv. 5 (2015) 92456-92472.

16. Shan, S.; Tian, Y. L.; Wang, S. H.; Wang, W. L.; Xu, Y. L.: (E)- $N^{\prime}-[1-$ (4-Aminophenyl)ethylidene]benzohydrazide. Acta Crystallogr. E64 (2008) 01363.

17. Shi, X. F.; Xing, Z. F.: (E)- $N^{\prime}$-[1-(4-Aminophenyl)ethylidene]-2hydroxybenzohydrazide methanol solvate. Acta Crystallogr. E63 (2007) 04771.

18. Yao, B. R.; Li, N.; Wang, C. H.; Hou, G. G.; Meng, Q. G.; Yan, K.: Novel asymmetric 3,5-bis(arylidene)piperidin-4-one derivatives: synthesis, crystal structures and cytotoxicity. Acta Crystallogr. C74 (2018) 659-665.

19. Li, N.; Bai, X. Y.; Zhang, L. S.; Hou, Y.: Synthesis, crystal structures and anti-inflammatory activity of four 3,5-bis(arylidene)$\mathrm{N}$-benzenesulfonyl-4-piperidone derivatives. Acta Crystallogr. C74 (2018) 1171-1179. 